\title{
Understanding the complexities of antibiotic prescribing behaviour in acute hospitals: a systematic review and meta- ethnography
}

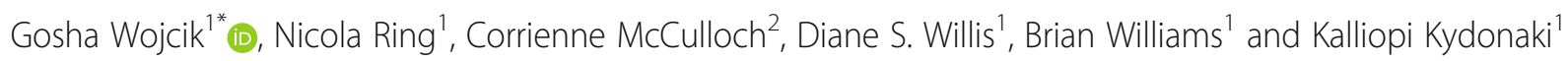

\begin{abstract}
Background: Antimicrobial resistance poses a serious global public health threat. Hospital misuse of antibiotics has contributed to this problem and evidence-based interventions are urgently needed to change inappropriate prescribing practices. This paper reports the first theoretical stage of a longer-term project to improve antibiotic prescribing in hospitals through design of an effective behaviour-change intervention.

Methods: Qualitative synthesis using meta-ethnography of primary studies reporting doctors' views and experiences of antibiotic prescribing in hospitals for example, their barriers to appropriate prescribing. Twenty electronic databases were systematically searched over a 10-year period and potential studies screened against eligibility criteria. Included studies were quality-appraised. Original participant quotes and author interpretations were extracted and coded thematically into NVivo. All study processes were conducted by two reviewers working independently with findings discussed with the wider team and key stakeholders. Studies were related by findings into clusters and translated reciprocally and refutationally to develop a new line-of-argument synthesis and conceptual model. Findings are reported using eMERGe guidance.
\end{abstract}

Results: Fifteen papers (13 studies) conducted between 2007 and 2017 reporting the experiences of 336 doctors of varying seniority working in acute hospitals across seven countries, were synthesised. Study findings related in four ways which collectively represented multiple challenges to appropriate antibiotic medical prescribing in hospitals: loss of ownership of prescribing decisions, tension between individual care and public health concerns, evidencebased practice versus bedside medicine, and diverse priorities between different clinical teams. The resulting new line-of-argument and conceptual model reflected how these challenges operated on both micro- and macro-level, highlighting key areas for improving current prescribing practice, such as creating feedback mechanisms, normalising input from other specialties and reducing variation in responsibility for antibiotic decisions.

\footnotetext{
* Correspondence: G.Wojcik@napier.ac.uk

${ }^{1}$ School of Health and Social Care, Edinburgh Napier University, EH11 4BN Edinburgh, UK

Full list of author information is available at the end of the article
}

(c) The Author(s). 2021 Open Access This article is licensed under a Creative Commons Attribution 4.0 International License, which permits use, sharing, adaptation, distribution and reproduction in any medium or format, as long as you give appropriate credit to the original author(s) and the source, provide a link to the Creative Commons licence, and indicate if changes were made. The images or other third party material in this article are included in the article's Creative Commons licence, unless indicated otherwise in a credit line to the material. If material is not included in the article's Creative Commons licence and your intended use is not permitted by statutory regulation or exceeds the permitted use, you will need to obtain permission directly from the copyright holder. To view a copy of this licence, visit http://creativecommons.org/licenses/by/4.0/ The Creative Commons Public Domain Dedication waiver (http://creativecommons.org/publicdomain/zero/1.0/) applies to the data made available in this article, unless otherwise stated in a credit line to the data. 
Conclusions: This first meta-ethnography of doctors` experiences of antibiotic prescribing in acute hospital settings has enabled development of a novel conceptual model enhancing understanding of appropriate antibiotic prescribing. That is, hospital antibiotic prescribing is a complex, context-dependent and dynamic process, entailing the balancing of many tensions. To change practice, comprehensive efforts are needed to manage failures in communication and information provision, promote distribution of responsibility for antibiotic decisions, and reduce fear of consequences from not prescribing.

Trial registration: PROSPERO registration: CRD42017073740.

Keywords: Antimicrobial resistance, Antibiotic decision-making, Prescribing behaviour, Doctors, Acute hospitals, Meta-ethnography, Qualitative synthesis

\section{Background}

The continuing emergence and spread of antimicrobial resistance (AMR) poses a major threat to public health and patient safety due to associated morbidity, mortality and healthcare expenditure [1]. The AMR crisis has been attributed, to a significant extent, to misuse and overuse of antibiotics [2,3]. A recent study looking at global antibiotic consumption, expressed in defined daily doses (DDD), found that it increased by $65 \%$ (21.1-34.8 billion DDDs) across 76 countries between 2000 and 2015 [4]. Although the increase was largely driven by low- and middle-income countries (LMICs), even within highincome countries, multidrug-resistant organism rates are rising. It is estimated that in the US and Europe alone, infections caused by antibiotic-resistant bacteria lead to at least 50,000 deaths annually, with hundreds of thousands more dying elsewhere across the world [2]. A simultaneous decline in new drug development by the pharmaceutical industry due to reduced financial inducements and challenging government regulatory mechanisms has further compounded the problem [5].

Rising resistance levels and unavailability of newer agents have led to coordinated efforts to implement new national and international initiatives, resume research efforts and minimise the use of currently available antibiotics to preserve their therapeutic effectiveness. These efforts have been underpinned by the concern that by 2050, AMR-related patient deaths will exceed 10 million annually worldwide, with a projected economic cost of 100 trillion US dollars [6]. Despite coordinated efforts, hospitals worldwide currently face significant problems with inappropriate antimicrobial use, as much as 30$50 \%$ of that usage being unnecessary or inappropriate, leading to worse health outcomes $[7,8]$. In the UK, despite some progress in primary care, a sustained reduction in total antibiotic prescribing in secondary care has not been observed. Whilst only $20 \%$ of antimicrobial consumption occurs in hospitals, the intensity of use is far higher than in the community. Evidence shows that hospital usage has increased by $6.3 \%$ over the last five years, despite widespread availability of local and national antibiotic prescribing recommendations [9]. This suggests that prescribing guidelines alone are insufficient to change practice and reduce the problem of AMR.

The need for well-designed antimicrobial stewardship (AMS) interventions has never been more critical. The Medical Research Council has long advocated the importance of identifying theory to understand the likely causal processes of change before undertaking the intervention effectiveness stage [10]. The assessment of the likely barriers and facilitators to inform the selection of intervention components is key to that process. However, systematic reviews of strategies employed to reduce inappropriate antibiotic use in hospitals have shown that behavioural and social influences remain underutilised in designing and evaluating AMS interventions [11]. The existing hospital AMS initiatives are not contextually designed or implemented with end-users of different specialties in mind [12]. As the majority of hospital antibiotic prescribing is currently performed by doctors, an in-depth exploration of the determinants that drive their behaviour within that context is crucial to changing that behaviour and enhancing the chances of planned interventions working in a real-world setting [13].

Although a recent Cochrane review provided recommendations on the effectiveness and safety of interventions to improve antibiotic prescribing to hospital inpatients [8], there remains a gap in the evidence-base on what behaviour change strategies work in hospitals, how to implement them and what refinements are needed to tailor the interventions to local contexts [14]. An exploration of the wide-ranging contextual, organisational and interpersonal determinants in antibiotic decision-making and their influence on different groups of prescribers has not received adequate attention.

The Cochrane Qualitative Implementation and Methods Group has increasingly recognised the importance of including qualitative findings within evidencebased healthcare research [15]. Qualitative research is particularly valuable in providing detailed descriptions of human thinking and behaviour in the contexts in which 
it occurs and capturing the depth and richness of people's views and experiences of, for example, delivering or receiving health interventions [16]. To date, some related qualitative syntheses have been conducted, such as in prescribing for respiratory infections in general practice [17] and in hospitals globally, including different groups of prescribers [18]. There is a large body of qualitative studies exploring hospital doctors ' antibiotic prescribing experiences, but this has not yet been systematically searched for and integrated within a robust qualitative synthesis. Yet, such research would enable knowledge gained from these hospital doctors' insights to generate new, clinically applicable theory to inform development of a much needed future behaviour change intervention.

\section{Methods}

This study aimed to identify and synthesise qualitative research reporting doctors 'views and experiences of the barriers and facilitators to appropriate antibiotic prescribing in acute hospitals and develop a conceptual model identifying how different pressures and dilemmas influence prescribing behaviour. Appropriate antibiotic prescribing was defined as the practice of initiation, monitoring, review and discontinuation of antibiotic therapy concordant with best practice such as guidelines.

\section{Study design}

There are various methods for synthesising qualitative research [19]. Noblit and Hare's meta-ethnography (ME) is an interpretive method widely used in health research as it systematically analyses multiple primary studies to go 'beyond' their participant and author findings to generate new conceptual models or theories through translation of original study findings reciprocally (accounts across studies are comparable) and refutationally (accounts contradict one another) to create a line-ofargument synthesis (where accounts can be drawn together in a new higher-level interpretation) [20, 21].

ME consists of seven overlapping phases: getting started; deciding what studies are relevant; reading studies; determining how studies relate; translating studies into one another; synthesising translations; and expressing the synthesis [21]. Reflecting the need for study transparency, ME methods and findings are reported according to the eMERGe guidance [22] and detailed in Additional file 1.

Phase 1 (getting started): ME was selected as the most suitable approach because of its ability to develop theory and/or conceptual understandings [21]. This approach was in line with this review's intention to identify components for a future antibiotic prescribing intervention. The study protocol was devised and registered with
PROSPERO (CRD42017073740). Ethical approval was not required.

\section{Search methods and study selection}

To help identify relevant studies (Phase 2), we used the SPIDER tool (Table 1) which facilitates searching of qualitative and mixed-method studies [23]. Our detailed search strategy is provided in Additional file 1. We systematically searched 20 electronic databases, including: EMBASE, MEDLINE, PubMed, ScienceDirect, Web of Science and ZETOC from 2007 to 2017. To maximise return, extensive search terminology and relevant synonyms were used, including medical subject headings $(\mathrm{MeSH})$, supplemented by free-text and broad-based terms.

Identification of qualitative research through electronic databases is challenging [24] so, the online search was supplemented with other methods, including handsearching of relevant publications, reference screening and citation searching of relevant reviews and included studies. Grey literature sources were searched for, including government reports, audits, conference proceedings and doctoral theses. Potential items for the ME were screened initially by title and abstract and then full text against our inclusion criteria (Table 2) by two reviewers (GW and $\mathrm{CM}$ ) working independently and then comparing outcomes. Any disagreements were referred to the full team for arbitration. Literature searching outcomes were reported using PRISMA (Fig. 1).

\section{Quality appraisal}

Phase 3 (reading included studies): each full-text article was read and re-read. Quality appraisal was conducted by two independent reviewers (GW and CM) based on the Critical Appraisal Skills Programme (CASP) assessment tool, which has been widely used in $\operatorname{ME}[25,26]$. Quality appraisal is not essential for ME, but it supports close reading of studies and helps assess each study's contribution to the final synthesis [20].

Data reporting original participant quotes (first-order) and author interpretations of participant data (secondorder) were extracted separately into NVivo v11 software. ME requires rich data for synthesis. If extraction revealed a lack of data, for example, few participant quotes, original authors were contacted for further data. If no further information was available, studies meeting our inclusion criteria but lacking data of suitable depth for synthesis were excluded, PRISMA updated (Fig. 1), and such papers were retained for later reflection (Phase $6)$.

\section{Analysis}

We adopted Toye et al.'s categorisation approach [27], including identifying concepts from qualitative studies, 
Table 1 Search terms identified using the SPIDER tool [23]
Sample (hospital clinicians)
Doctor* OR physician* OR clinician* OR medical staff OR health personnel
Phenomenon of Interest (antibiotic
Antibiotic prescribing OR overprescribing OR misuse $O R$ overuse $O R$ antibiotic stewardship $O R$ prescribing in acute hospitals) resistance $O R$ guideline adherence OR decision-making OR practice behaviour AND hospita* OR acute care OR hospital ward
Design/ Evaluation/ Research type Qualitative OR focus group* OR interview* OR ethnograph* OR observation* ( ${ }^{*}$ qualitative)

See Additional file 1 for Hybrid Qualitative Filters

grouping concepts into higher conceptual categories, further re-grouping categories into overarching themes and, developing a line-of-argument that makes sense of the themes. Through constant comparison, studies were first related by findings (Phase 4) to identify 'concepts' (key metaphors, phrases and meaningful ideas), the raw data of ME [26], to see how studies compared or not. This was done using the first- and second-order data extracted into NVivo and then organised using Microsoft Excel spreadsheets. As the papers were re-read, additional ideas that arose were noted. Studies were initially grouped by their primary thematic focus into two clusters and then data across the studies were ordered into larger related categories. Continual reference throughout to original studies and conservation of their unique language/terms was critical. The balancing of the developed inter-related larger categories and understanding the way they are influenced by other factors was central to translating the studies into one another, the next stage of ME.

Phase 4 seamlessly led into the analytical process of study translation (Phase 5). Through discussion, similar reported concepts were merged and collapsed into higher conceptual categories pertaining to the same aspects of antibiotic prescribing. First- and second-order constructs (participants' quotes and authors' interpretations) in each study were continuously compared with those in other studies. We used a 'hands on ' approach, drawing arrows, lines, creating concept maps and matrices. This process was idiomatic and carried out chronologically, starting from the earliest publication. We compared key concepts from paper one with paper two, synthesised them and compared the outcome with paper three, and so on. The interpretations and explanations provided by the original study authors were subsequently compared and translated across papers to achieve a synthesis. Translation of findings was reciprocal where similar concepts (albeit expressed differently) were drawn together and refutational, where contradictory or disconfirming concepts were noted. Where differences were noted, for example, if a study reported different concepts from the others, we returned to full text papers to understand its context, such as whether participants were in a different setting or of different gender. The expanded groupings were then refined and re-arranged for two clusters of studies, first separately and then drawn together until they were considered to explicitly and precisely reflect the synthesised findings. This process enabled us to 'go beyond findings from individual studies, from simple descriptions of the data to developing third-order interpretations [28]. Translation led to the development of overarching themes.

Phase 5 merged into Phase 6 (synthesis of translations), whereby through reflection and discussion we went over and above the developed themes to create a new line-of-argument (LOA), that is our 'third-order constructs interpretation', a picture of the findings built on the individual parts of studies [20]. Findings generated during the translation, created spreadsheets, data matrices and our explanations and interpretations provided the foundation for higher analysis. Themes were brought together and matched against original author

Table 2 Study inclusion and exclusion criteria

\footnotetext{
Inclusion criteria

- Primary research studies reporting doctors' views and experiences of antimicrobial prescribing in acute hospital settings, including adult and paediatrics

- Used qualitative methods of data collection (e.g., interviews, focus groups) and inductive analysis (e.g., grounded theory, phenomenological analysis)

- Mixed-methods studies only if the qualitative data are discreet and findings reported adequately

- Studies carried out in countries considered to have a developed

healthcare system according to international classification

- Published in English language between 2007 and 2017
}

\section{Exclusion criteria}

- Primary research reporting doctors` views and experiences of prescribing other treatments or other aspects of prescribing e.g., costs, effectiveness.

- Research on prescribing antibiotics in other settings e.g., primary care or residential settings

- Studies conducted in countries not considered to have a developed health care system ${ }^{\text {a }}$

- Sample including prescribers other than acute hospital doctors e.g., general practitioners or nurses

- Studies that did not report primary qualitative data collection and analyses e.g., quantitative research, descriptive case studies, commentaries, editorials, reviews. Mixed-methods studies where qualitative data were not reported separately 


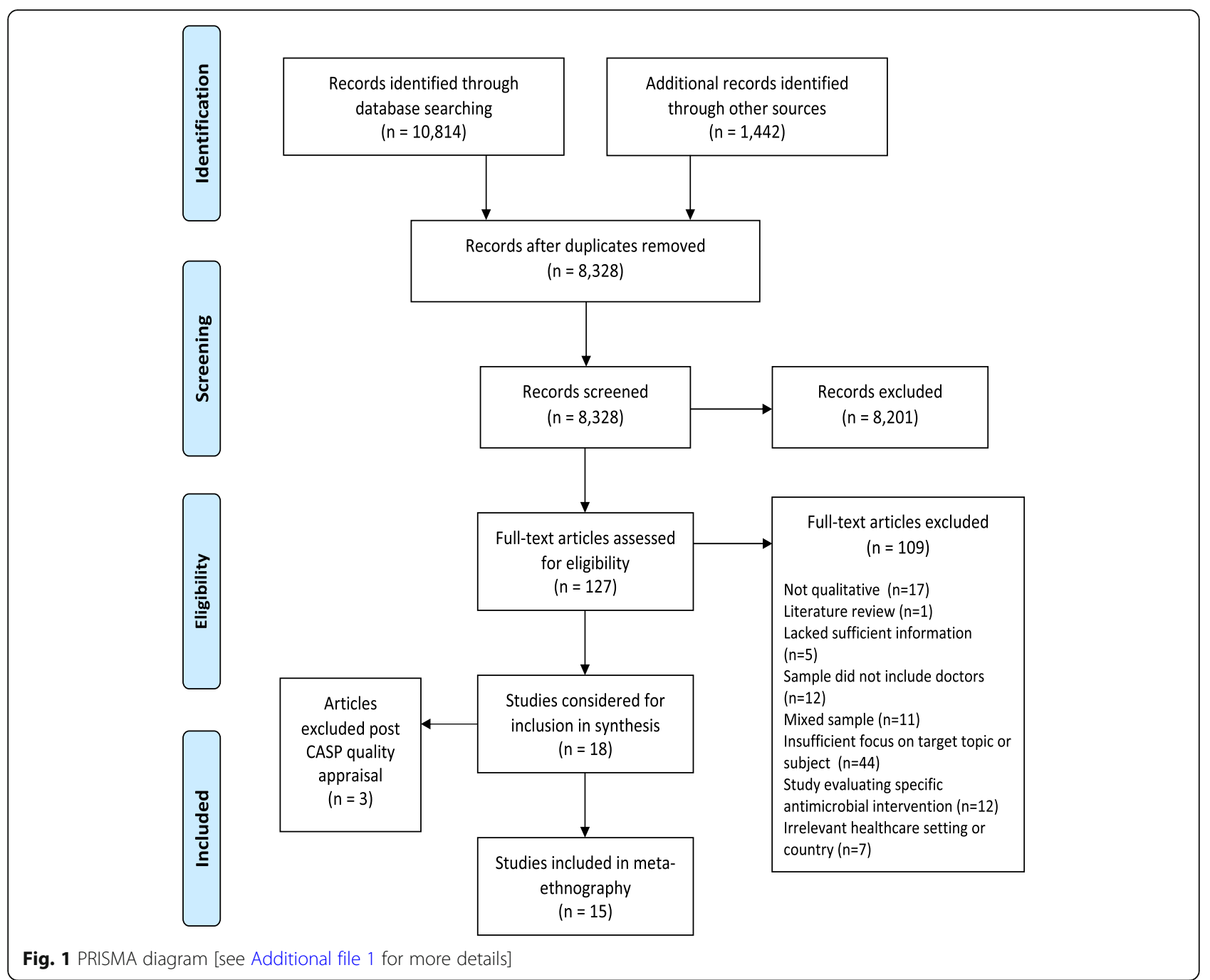

interpretations and participant quotes from each study to create a new LOA.

Reflection is critical in ME and this was achieved in three ways [26]: team discussions to check accuracy and emerging findings/perspectives; three group consultations across the study with key stakeholders (professionals involved in hospital antimicrobial stewardship and health service users) and, comparing our LOA with findings in studies excluded following quality appraisal to determine whether their inclusion would have altered our final synthesis. Overall, these processes enabled us to reflect on and refine our LOA and propose a new conceptual model of the multi-dimensional nature of medical antibiotic prescribing which we then expressed in our synthesis findings using narrative and visual representation (Phase 7).

\section{Results}

Overall, 12,256 possible references were identified (Fig. 1). Eighteen qualitative papers met our inclusion criteria. Following quality appraisal (Table 3), three papers were excluded due to low quality of their reporting [29-31]. Fifteen papers reporting findings from thirteen studies were finally included in the synthesis [32-46].

The included studies were from seven countries across three continents: Australia [34, 42, 45, 46], USA [36-38] and Europe, including the UK [35, 40, 41, 44], Belgium [32], Sweden [33], Switzerland [43] and Norway [39]. Studies were conducted in 43 acute hospitals, including regional, metropolitan, tertiary and secondary care. All included studies involved research carried out in public hospitals and four papers drew the sample from a mix of hospitals (i.e., public, private and federal) [36-39]. Thirteen papers described the hospitals as teaching [32, 3537, 39-46]. The studies reported the experience of 336 doctors practising across various disciplines from a range of medical and surgical fields. All except three studies $[36,43,44]$ provided gender information that included 274 participants, from which 106 (39\%) were women. Not all authors provided details of their study 
context (i.e., hospital type) and it was not always possible to determine participants 'ethnicity, specialty, length of clinical experience, and exact area of medical expertise. Most studies specified participants` level of experience representing a range of seniority $(n=14)$, whilst one study focused specifically on junior (foundation year) doctors [35]. The age of participants ranged between 20 [35] and 70 years [33].

Sample size varied considerably, from 10 [46] to 64 doctors [45]. Data were collected using individual interviews $(n=13)$ [33-35, 37-46], focus groups $(n=1)$ [32] and a mixed-methods approach comprising an online survey and semi-structured interviews followed by an observational study $(n=1)$ [36]. Overall, the studies had an acceptable methodological quality. However, most studies neglected the value of reflexivity $(n=12)$, with only three studies reporting how the authors' social background, location, role, and assumptions may have affected the research process and findings [39, 44, 46].

Characteristics of the 15 papers, including author, year of publication, country/setting, study focus, population, data collection, analytic approach and key findings, are detailed in Table 4.

Studies related (Phase 4) by their focus into two clusters:

- Cluster A - studies that focused on the adherence to antimicrobial guidelines, including the barriers and enablers to uptake and the suboptimal use [32, $38,46]$.

- Cluster B - studies describing the experience of antibiotic prescribing with differing levels of emphasis placed on the influences on the prescribers` behaviour, ranging from the drivers of antibiotics prescribing, clinical decision-making to awareness of AMR [33-37, 39-45].

Across clusters A and B, 142 concepts emerged with the resulting 17 higher conceptual categories (HCCs) or 'piles' that shared meaning. The reported concepts within each conceptual category are detailed in Additional file 2. From these concepts and HCC, four overarching themes were identified during study translation (Phase 5): (1) Loss of ownership of prescribing decisions, (2) Tension between individual care and broader public health concerns, (3) Evidence-based practice versus bedside medicine, and (4) Diverse priorities between different clinical teams. Themes $1-3$ were derived from reciprocal translation (findings were compatible). Theme 4 arose from refutational analysis when it was noted that some translated findings described alternative dissonant perspectives of the same phenomenon. Themes are presented below with narrative exemplars in Additional file 3.

\section{'Loss of ownership of prescribing decisions'}

Many hospital healthcare professionals have a role in antimicrobial stewardship but overall responsibility for antibiotic decisions lies with prescribing clinicians. Many decisions are made by senior clinicians and then enacted by junior doctors. However, during nights and weekends, this arrangement shifts, and junior doctors are often expected to manage complex cases alone and make decisions to prescribe antibiotics on behalf of their senior colleagues, with limited support and feedback available at the time [34-40, 42, 44, 46].

When care delivery happens 'out-of-hours', the allocation of prescribing responsibility becomes ambiguous. Although junior doctors are expected to initiate or escalate antibiotics, they are hesitant to question or change decisions of their senior colleagues consequently reporting feelings of disempowerment [35, 41, 42, 44]. Deescalating or stopping treatments is considered a senior medical decision-maker role as this requires professional confidence and experienced clinical judgement. Making an independent clinical judgement is viewed by less experienced doctors as unrealistic, or 'something of a dark art' [42], highlighting variation in the perceived responsibility for prescribing decisions.

Patients transitioning between hospital wards means that the provision of care takes place in multiple hospital locations and across various professional groups, adding to the complexity. Doctors' rotations, rapid ward rounds, numbers of staff delivering care and patients being cared for 'remotely' from their primary medical team compounds the problem, leading to frustration, anxiety and ultimately distancing from engaging with decision-making [33, 35-37, 39, 40, 43, 44]. Lack of awareness of what ultimately happens to the patient and whether the prescribed antibiotic therapy was the correct choice for the patient denies junior doctors the opportunity to learn from occasions when their prescribing decisions had been over-ruled or changed.

There was also a concern that some information handed over to the next shift (or clinical area) is not always acted on and prescribed antibiotics are not reviewed by the subsequent clinical team taking over a patient's care. Fast-paced clinical environments, errorprone handovers, disjointed information, and cumbersome IT systems present further challenges [32-37, 39, $40,42-44,46]$. Three studies highlighted that poor documentation of decisions and inconsistencies in monitoring and treatment plans compounded the problem and created a sense of anonymity or 'invisibility' of decisions $[35,41,44]$. When reasons for antibiotic prescriptions in clinical patient notes are not documented, clear or easy-to-find, clinicians have to guess whether initial decisions regarding antibiotic choice and rationale was accurate and justified. This incomplete patient 


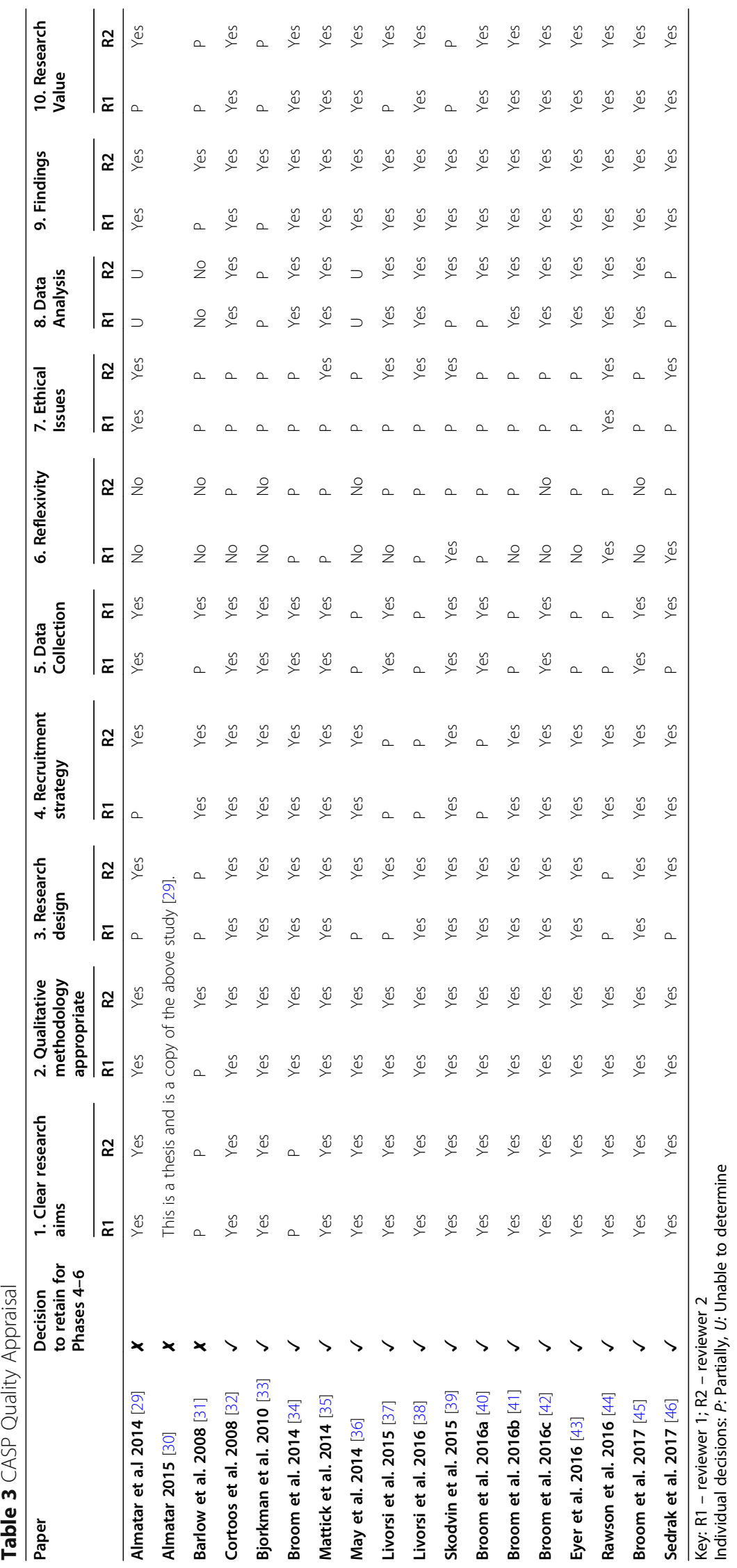


Table 4 Summary of qualitative papers included in the synthesis

\begin{tabular}{|c|c|c|c|c|}
\hline Study & $\operatorname{Aim}(s)$ & Sample & $\begin{array}{l}\text { Data collection \& } \\
\text { analysis }\end{array}$ & Key findings \\
\hline $\begin{array}{l}\text { Cortoos et } \\
\text { al. } 2008 \\
\text { [32] }\end{array}$ & $\begin{array}{l}\text { To determine the opinions and } \\
\text { problems concerning the use of a } \\
\text { local antibiotic hospital guideline. }\end{array}$ & $\begin{array}{l}1 \text { public tertiary care university } \\
\text { teaching hospital }\end{array}$ & Focus Groups & 7 themes reported: \\
\hline \multirow[t]{2}{*}{ Belgium } & & $\begin{array}{l}22 \text { physicians from internal medicine } \\
\text { ( } 7 \text { residents / } 6 \text { staff) and surgery ( } 6 \\
\text { residents/ } 3 \text { staff). }\end{array}$ & Framework Analysis & \multirow{2}{*}{$\begin{array}{l}\text { General attitudes and guideline } \\
\text { interpretation; } \\
\text { guideline familiarity and awareness; } \\
\text { guideline contents and agreement; } \\
\text { social influence; multidisciplinary } \\
\text { approach, organizational constraints; } \\
\text { attitudes about specific } \\
\text { interventions. }\end{array}$} \\
\hline & & Ages: 26-60, 5 females/17 males. & & \\
\hline
\end{tabular}

Bjorkman To explore and describe perceptions 7 acute public hospitals

et al. 2010 of antibiotic prescribing among

[33] Swedish hospital physicians.

Sweden

Broom et To investigate the experiences of al. 2014 doctors who prescribe antibiotics.

[34]

Australia

Mattick et

al. 2014

[35]

UK

(England \&

Scotland)

May et al. To explore current practices and 2014 [36] decision-making regarding

Livorsi et

al. 2015

[37]

USA antimicrobial prescribing among Emergency Department (ED) clinical clinicians.

To explore the antimicrobial prescribing experiences of foundation year (FY) doctors.

To understand the professional and psychological factors that influence physician antibiotic prescribing habits in the inpatient setting.
20 hospital physicians (5 urology physicians, 5 from surgery, 10 from internal medicine).

Ages: 31-70, 5 females/15 males.

1 acute regional public hospital

30 doctors from: emergency medicine (3), general medicine (4), geriatrics (3), intensive care (2), obstetrics and gynaecology (3), oncology (2), orthopaedics (2), paediatrics (1), renal medicine (2), sexual health (1), surgery (2), urology (1) and infectious diseases (4). House officers (4), registrars (7), advanced trainees (2), consultants/ staff specialists (11), consultants/ senior staff specialists (5).

9 females/21 males.

2 public secondary care teaching hospitals

33 junior doctors (21 FY1 and 12 FY2) working in medical and surgical wards.

Ages: 20-35, 18 females/15 males

8 acute hospitals, including: 5 private (2 tertiary care and 3 tertiary academic centres), 2 federal and 1 public

21 clinicians (attending physicians, residents, and mid-level clinicians with at least 2 years of ED experience).

No gender documented.

2 acute teaching hospitals (1 public tertiary care and 1 federal)

30 inpatient physicians: 10 physicians-in-training (8 internal medicine, 2 internal medicine/ paediatrics) \& 20 supervisory staff (17
Semi-structured Interviews

Phenomenographic Analysis

Semi-structured Interviews

Thematic Analysis

Narrative Interviews

Framework Analysis

Semi-structured Interviews (mixedmethods study)

Thematic Analysis

Semi-structured Interviews

Thematic Analysis
6 overarching themes reported:

5 main categories of perceptions of hospital antibiotic prescribing and AMR:

Prefer "effective" treatment; too uncertain to be restrictive; stuck in the healthcare system; aware and restrictive, but support required; aware, interested and competent.

6 main themes reported:

Everyday sensitivity toward resistance; risk, fear and uncertainty; time, pressure and uncertainty; benevolence and the emotional prerogative; habitus and the internalisation of peer practice norms; hierarchies and the localisation of antibiotic prescribing.

Personal incident narratives about antimicrobial prescribing; antimicrobial prescribing experiences; systems issues; working relations; educational experiences and needs; process-related data.

5 overarching themes reported:

Resource and environmental factors that affect care; access to and quality of care received outside of the ED consult; patient-provider relationship; clinical inertia; local knowledge generation

4 themes reported:

Antibiotic over-use is recognised but generally accepted; the potential adverse effects of antibiotics have a limited influence 
Table 4 Summary of qualitative papers included in the synthesis (Continued)

\begin{tabular}{ll}
\hline Study Aim(s) & Sample \\
\hline & hospital medicine, 3 pulmonary/ \\
& critical care). \\
& 10 female/20 males
\end{tabular}

Livorsi et To assess physician knowledge and al. 2016

[38]

USA guidelines through the use of case vignettes.
Skodvin et To investigate factors influencing al. 2015

[39] antimicrobial prescribing practices among hospital doctors.

Norway

Broom et

To identify why inappropriate

al. 2016a

[40]

UK

Broom et

al. $2016 b$

[41]

UK prescribing trends continue.

To explore doctors' experiences of antibiotic prescribing, and the role of social and institutional factors in influencing the decision-making process.
20 doctors: 8 consultant, 12 non-
2 acute teaching hospitals (1 public tertiary care and 1 federal)

30 inpatient physicians: 10 physicians-in-training (8 internal medicine, 2 internal medicine/ paediatrics) \& 20 supervisory staff (17 hospital medicine, 3 pulmonary/ critical care).

10 female/20 males

12 public and 1 private hospitals (3 teaching and 10 non-teaching)

15 doctors from five major medical fields (internal medicine (4), surgery (4), infectious diseases specialists (2), other medical field: oncology, neurology and intensive care), Interns/residents/consultants 2/5/8.

Ages: 25-65, 8 females/7 males.

1 public teaching hospital

20 doctors: 8 consultant, 12 nonconsultants from medical (15) and surgical specialty (5).

9 females $/ 11$ males.

1 public teaching hospital consultants from medical (15) and surgical specialty (5).

9 females $/ 11$ males.

1 public regional teaching hospital

30 doctors from emergency medicine (3), general medicine (4), geriatrics (3), intensive care (2), obstetrics and gynaecology (3), oncology (2), orthopaedics (2), paediatrics (1), renal medicine (2), sexual health (1), surgery (2), urology (1) and infectious diseases (4). Sample included house officers, registrars, advanced trainees, consultants/staff specialists and consultants/senior staff specialists.

\section{Data collection \& Key findings}

analysis

Semi-structured Interviews

Thematic Analysis

Semi-structured Interviews

Thematic Analysis

Analis

on physicians' decision-making; physicians-in-training are strongly influenced by the antibiotic prescribing behaviour of their supervisors; reluctance to provide critique, feedback or advice.

3 major themes reported:

Lack of awareness of specific guideline recommendations; tension between adhering to guidelines and the desire to individualise patient care; scepticism of certain guideline recommendations.

6 major themes reported:

Colleagues; microbiology; national guideline; training; patient assessment; leadership.
Semi-structured Interviews

Framework Analysis

Semi-structured Interviews

Framework Analysis

(1)

Negotiating multiple masters; junior doctors 'stuck in the middle' between infectious diseases, clinical microbiology and their supervising team; the dynamics of laboratory vs clinical medicine; the transmission of habit: evidence confronts mentoring, anecdote and experiential learning.

Semi-structured Interviews

4 main themes reported:

Thematic Analysis

Contesting 'best' practice: risk and ambivalence; 'fear of losing them' and the role of patient vulnerability; intra-professional and workplace context; 'craft groups' and the perpetuation of localised norms. 
Table 4 Summary of qualitative papers included in the synthesis (Continued)

\begin{tabular}{|c|c|c|c|c|}
\hline Study & $\operatorname{Aim}(s)$ & Sample & $\begin{array}{l}\text { Data collection \& } \\
\text { analysis }\end{array}$ & Key findings \\
\hline & & 9 females /21 males. & & \\
\hline $\begin{array}{l}\text { Eyer et al. } \\
2016 \text { [43] }\end{array}$ & \multirow{3}{*}{$\begin{array}{l}\text { To determine reasons for using } \\
\text { antibiotics to treat asymptomatic } \\
\text { bacteruria in the absence of a } \\
\text { treatment indication. }\end{array}$} & $\begin{array}{l}1 \text { public tertiary care university } \\
\text { teaching hospital }\end{array}$ & $\begin{array}{l}\text { Semi-structured } \\
\text { Interviews }\end{array}$ & 5 main themes reported: \\
\hline \multirow[t]{2}{*}{ Switzerland } & & $\begin{array}{l}21 \text { general medicine physicians: } 12 \\
\text { residents/9 senior physicians. }\end{array}$ & \multirow[t]{2}{*}{ Thematic Analysis } & \multirow{2}{*}{$\begin{array}{l}\text { Treatment of laboratory results } \\
\text { without considering the clinical } \\
\text { picture; physician-centred factors; } \\
\text { external factors; lack of attention to } \\
\text { detail or analytical thinking, } \\
\text { particularly under time constraints; } \\
\text { overtreatment due to trivialization } \\
\text { of urinary tract infection. }\end{array}$} \\
\hline & & No gender documented. & & \\
\hline $\begin{array}{l}\text { Rawson et } \\
\text { al. } 2016 \\
{[44]}\end{array}$ & $\begin{array}{l}\text { To map out and compare the } \\
\text { decision-making processes } \\
\text { employed for acute infection }\end{array}$ & $\begin{array}{l}3 \text { public university teaching hospitals } \\
\text { (mix of secondary and tertiary care } \\
\text { providers) }\end{array}$ & $\begin{array}{l}\text { Semi-structured } \\
\text { Interviews }\end{array}$ & 3 overarching themes reported: \\
\hline \multirow[t]{2}{*}{ UK } & $\begin{array}{l}\text { management on the hospital wards } \\
\text { by non-infection medical specialties } \\
\text { and explore any factors that } \\
\text { influenced this process. }\end{array}$ & $\begin{array}{l}20 \text { physicians ( } 9 \text { consultants, } 4 \\
\text { registrars, } 2 \text { trainees, } 5 \text { junior } \\
\text { doctors) from non-infection medical } \\
\text { specialties (general internal } \\
\text { medicine, such as cardiology, } \\
\text { respiratory, and geriatric medicine) } \\
\text { and augmented care specialties } \\
\text { (haematology and nephrology). }\end{array}$ & \multirow[t]{2}{*}{ Grounded Theory } & \multirow[t]{2}{*}{$\begin{array}{l}\text { Mapping the decision-making } \\
\text { process; factors influencing the } \\
\text { decision-making process; windows } \\
\text { of influence on decision making. }\end{array}$} \\
\hline & & No gender documented. & & \\
\hline $\begin{array}{l}\text { Broom et } \\
\text { al. } 2017 \\
{[45]}\end{array}$ & $\begin{array}{l}\text { To examine how hospital doctors } \\
\text { balance competing concerns around } \\
\text { antibiotic use and resistance. }\end{array}$ & $\begin{array}{l}2 \text { acute public teaching hospitals ( } 1 \\
\text { regional and } 1 \text { metropolitan) }\end{array}$ & $\begin{array}{l}\text { Semi-structured } \\
\text { Interviews }\end{array}$ & 2 key themes: \\
\hline \multirow[t]{3}{*}{ Australia } & & $\begin{array}{l}64 \text { doctors from anaesthetics, } \\
\text { emergency, geriatrics, gynaecology, } \\
\text { haematology, ICU, infectious } \\
\text { diseases, nephrology, oncology, } \\
\text { orthopaedics, paediatrics, palliative } \\
\text { care, respiratory, sexual health, and } \\
\text { surgery. }\end{array}$ & \multirow[t]{3}{*}{ Framework Analysis } & \multirow[t]{3}{*}{$\begin{array}{l}\text { The significance of resistance for the } \\
\text { hospital and the role of doctor in } \\
\text { perpetuating resistance; } \\
\text { overprescribing; easier and without } \\
\text { perceived immediate risk. }\end{array}$} \\
\hline & & 27 junior doctors, 37consultants. & & \\
\hline & & 28 females/36 males. & & \\
\hline $\begin{array}{l}\text { Sedrak et } \\
\text { al. } 2017 \\
{[46]}\end{array}$ & \multirow{3}{*}{$\begin{array}{l}\text { To elucidate potential barriers and } \\
\text { enablers to the adherence to } \\
\text { antibiotic guidelines by clinicians } \\
\text { treating community-acquired } \\
\text { pneumonia. }\end{array}$} & 1 public tertiary teaching hospital & $\begin{array}{l}\text { Semi-structured } \\
\text { Interviews }\end{array}$ & 3 main categories reported: \\
\hline \multirow[t]{2}{*}{ Australia } & & $\begin{array}{l}10 \text { clinicians from emergency } \\
\text { medicine (4), general medicine (4) } \\
\text { and infectious disease (2). } 5 \\
\text { registrars and } 5 \text { consultants. }\end{array}$ & \multirow[t]{2}{*}{ Thematic Analysis } & \multirow{2}{*}{$\begin{array}{l}\text { Knowledge, including familiarity } \\
\text { with guidelines; attitudes, including } \\
\text { confidence in antibiotic guidelines; } \\
\text { behaviour, including documentation } \\
\text { and communication, experience and } \\
\text { clinical judgement. }\end{array}$} \\
\hline & & 5 females/5 males. & & \\
\hline
\end{tabular}

information impacts on clinicians` ability to take ownership of antibiotic prescribing decisions.

\section{'Tension between individual care and broader public health concerns'}

In uncertain clinical situations, doctors must make decisions in the presence of multiple and often conflicting objectives. While the ethical principle of a 'good doctor' is to make decisions based on what is best for the individual patient [34], at the same time, clinicians have a responsibility to consider population- level consequences of overprescribing. On one hand, antibiotic overprescribing is recognised as a serious global concern but, on the other hand, not treating an infection may lead to serious patient complications, even death [33, 34, 37, 40, 42-45], and loss of professional reputation. The abstract reality of future AMR causes internal conflict for the treating clinician facing the concrete reality of the 'here and now' - the patient's clinical status and perhaps pressure from family and patients to 'do something'. The short-term individual costs (for patients and professionals) have 
to be constantly weighed up against longer-terms societal gains.

Although clinicians consider AMR and its potentially severe consequences when choosing treatment, the threat of resistance is generally perceived to be a distant or not immediate issue [33, 34, 37, 42, 45]. With the exception of clinicians working within infectious diseases and microbiology departments [33], most participants appeared to downgrade the importance of the problem and its potentially devastating consequences during their prescribing decision-making process. Long-term effects of resistance at the wider community-level are not prioritised, and some degree of overuse of antibiotics to manage immediate patient risks is considered to be allowed and socially acceptable [33, 34, 37, 40, 45].

The risks of over-prescribing to the individual patient tend to be disregarded [45]. Some clinicians consider antibiotics a 'peripheral thing', of 'limited concern' [34] with the threat of AMR as a theoretical problem, which is morally and professionally important but not necessarily practical $[33,37,40,42,45]$. Recognition that individual practice contributes to the emergence of AMR is generally low and some clinicians are 'desensitised' to the problem [45]. Absence of feedback on juniors` antibiotic prescribing limits the opportunity to identify reasons for the knowledge deficits and improve prescribing practice.

\section{'Evidence-based practice versus bedside medicine'}

Internal reasoning, or the way clinicians make sense of their decisions, plays a significant role in antibiotic prescribing. Prescribing behaviour, which may at first appear as 'non-rational' or at odds with the evidence, is in fact a realistic and logical choice at the bedside, where positive patient outcomes, maintaining professional reputation and approval from supervisors take a priority [33-37, 41-45]. The health of individual patients lies at the core of medical professionalism and forms part of their professional identity. Being seen by the patient and/or relatives to be 'doing good' drives clinicians to prescribe antibiotics for their patient regardless of whether it is evidence-based or not [44]. This internalised logic of over-prescribing is driven by the desire to improve patient condition(s) or at least provide a 'beacon of hope ' [43]. This rationale interplays with the expectations of never missing a diagnosis. Prescribing antibiotic treatment is seen a confirmation that 'at least something has been done` [38].

In busy hospital environments, professional competence is being constantly evaluated. Decisions about whether to prescribe antibiotics are heavily influenced by fear of consequences for prescribers. Missing a potentially treatable infection could result in serious patient harm. Administering antibiotics or prolonging their use creates a perception of an emotional safety net [33, 34, 36-40, 42-46]. Although experience helps to identify and treat the severely ill patients, 'erring on the side of caution' and prescribing antibiotics 'just in case' provides reassurance and is therefore the default option irrespective of grade or experience [37].

Junior doctors report experiences of being criticised and seen by colleagues as incompetent when deciding not to treat $[35,37,40,42,45]$; in contrast, conservative antibiotic decision-making is rarely recognised as good practice [43]. Senior doctors' preferences, expectations and prescribing habits also influence junior doctors' prescribing decisions. Junior doctors risk facing social disapproval if their decision not to prescribe is at odds with the `social norms` of the hospital [34].

Patient demand, expectations of patients' families and the developing 'consumerism culture' pose additional pressure [40], resulting in a low threshold for prescribing antibiotics, [33, 34, 36, 37, 39, 41-43, 45, 46]. Fear of patient complaints and of potential lawsuit drives clinicians to adopt defensive medicine approaches and prescribe broad-spectrum antibiotics unnecessarily, irrespective of the healthcare system they work in (public or private). However, external factors, such as patient access to care in the private health system, hinder doctors' ability to foster AMS. For instance, in the US, the emergency departments disproportionately provide care to lowincome and uninsured patients [36]. As a result, doctors must not only account for the clinical scenario but also consider the patient's ability to obtain follow-up care.

Prescribing according to guidelines offers some reassurance and protection, provided these are evidencebased, up-to-date, easily available, and accessible and that doctors have time to consult them [32-34, 37-39, 41, 43, 44, 46]. Digressing from antibiotic guidelines is rationalised by the potential discrepancies between guidelines and practice. When the individual case of a patient does not 'fit' readily into guidelines, clinical judgement must be applied [32, 35, 38, 41, 46].

\section{'Diverse priorities between different clinical teams'}

Multidisciplinary input is essential during hospital inpatient care. However, a multitude of experts are involved in patient care, with different tasks or interventions performed by different professionals, who may have different goals for the patient, which can result in variation of care, including antibiotic use. For instance, diverse priorities are evident in the weighting given to different phases of the antibiotic decision-making process between speciality groups. Despite a common overall approach, emergency department (ED) clinicians and surgical specialities emphasise immediate patient care and infection prevention including initiating antibiotics [32, 36, 40, 45, 46], whilst medical specialities focus 
on longer-term infection management concerns, including refining/reviewing of initial prescribing decisions and stopping antibiotics [41, 43, 44].

Heightened awareness of sepsis and associated risks and complications culminates in an urgency for surgeons and ED clinicians to commence antibiotics as soon as possible in anyone suspected of having an infection [36, 39]. By contrast, acute care medicine doctors report a common stepwise approach to the decision process surrounding acute infection management, whereby new information is constantly considered in the context of prior knowledge [44] and the use of microbiology test results when selecting antimicrobial therapy is emphasised. Within the same hospital, different clinical teams can have diverging opinions on, and requirements from, guideline content. For example, whilst surgical groups describe a strict interpretation of antibiotic guidelines [32], internal medicine doctors highlight that guidelines are incomplete by promoting a standardised, 'one-size fits all' approach to antibiotic prescribing [36, 38, 39, 41, 44, 46].

Most clinicians (both genders, across settings and healthcare sectors) recognise the benefits of collaboration, including the availability of a second opinion in the treatment of infections and the support for the improved use of antibiotic prescribing guidelines. However, junior doctors experience difficulties in negotiating prescribing decisions with multiple authoritative figures from across various clinical teams [34, 35, 41, 44]. Effective collaboration and senior support were perceived by junior doctors as key facilitators in remedying deficiencies in practical knowledge of appropriate antibiotic prescribing [33-35, 38-40, 42-44].

Key professional collaborators identified in antibiotic prescribing were microbiology, infectious disease specialists and pharmacy. Infection diseases specialists were recognised as helping hospital doctors in AMR prevention by promoting and encouraging the use of guidelines and appropriate narrow-spectrum antimicrobials during handover meetings and ward rounds [33, 39]. Clinical microbiology colleagues were reported as acting as an important communication channel in infection management $[35,39,44]$. Medical doctors especially described their services and advice as valuable and convenient to access. Although these experts were generally highly approved across medical and surgical fields, the relationship with them varied significantly depending on individual clinicians` interest in infectious diseases [32, 33, $35,41,45]$.

The presence of ward clinical pharmacists generated conflicting opinions. Most clinicians from medical and surgical groups (mostly males representing different levels of seniority) described pharmacists as helpful in discussing and sharing rationales for antibiotic prescriptions and prompting antibiotic review and deescalation [37, 40,44]. However, they were perceived by some participants (mostly male physicians from internal medicine) as interference [32].

\section{Line-of-argument synthesis}

From translation of findings across the 15 studies, a new line-of-argument emerged. This final stage in the process of meta-ethnographic analysis (Phase 6) enabled us to develop a higher order interpretation, that is, to generate a conceptual model drawn from, 'but more than the sum of ', the final themes [21]. Through team reflection and by revisiting the original studies, it gradually became apparent that the four overarching themes overlapped and a more complex nuanced interaction between two micro- and macro-level dimensions of hospital antibiotic prescribing emerged. These two dimensions constantly and simultaneously interacted with each other producing multiple tensions for prescribers and formed the basis for our conceptual model (Fig. 2).

The model illustrates the multidimensional nature of hospital antibiotic decision-making and reflects the array of pressures and dilemmas which need to be balanced by clinicians as they decide their prescribing action(s). This multidimensional nature of antibiotic decision-making describes a complex dynamic and for every clinician, there will be a degree of interdependence between different factors influencing prescribing practice, depending on their level of expertise and ability to tolerate risks for their patient and themselves. The illustrated elements, or factors, will form independent components on one level. However, they are not separate or discreet but constitute an integral part of a whole and will therefore exert a degree of direct or indirect influence on prescribing decisions. These elements coexist, interact, and create a constant dynamic. Both macro (wider social structures, including the norms, standards, social and organisational constraints for human behaviour) and micro (individual behaviours) dimensions feature a complex interplay of influence, authority, and the pursuit of treatment goals. The macro-level structures of hospitals provide the social and cultural setting for healthcare professionals to relate to each other, constantly shaping and influencing micro-level dimensions that drives individual behaviours and everyday practice.

This unique and evolving dynamic results in the creation of micro-structures of influence, such as internalised logic of prescribing that underpins antibiotic use and drives social interaction with colleagues and patients. An understanding of these contextual drivers of overuse on both macro- and micro-level is fundamental to the development of sustainable interventions to optimise antibiotic use by hospital doctors. 


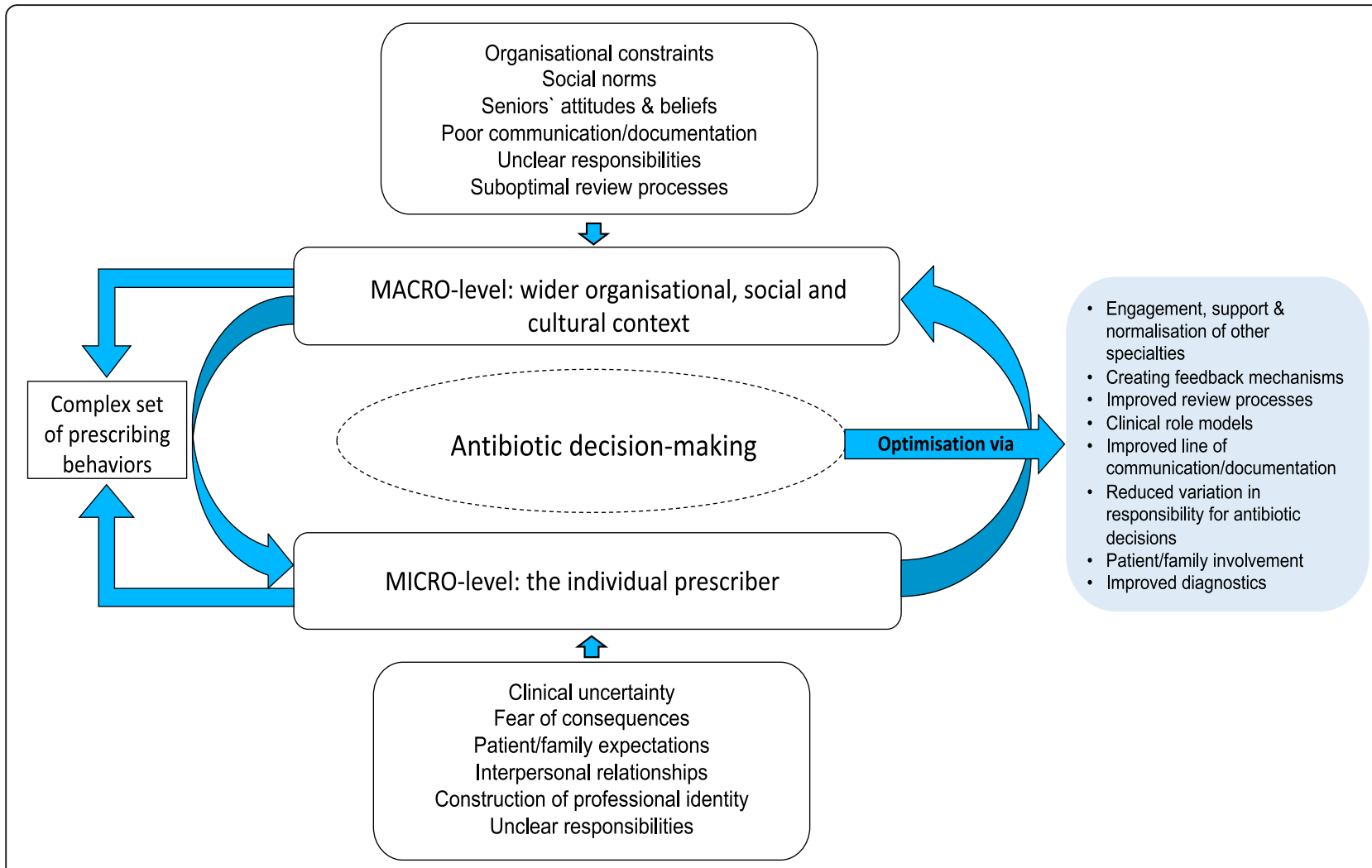

Fig. 2 Conceptual model depicting multidimensional nature of antibiotic prescribing in hospital settings

\section{Discussion}

This review is the first to apply an interpretive metaethnographic approach and propose a conceptual model to understand the nature of antibiotic prescribing in acute hospitals. The exploration of the challenges to appropriate prescribing in hospitals revealed tensions and uncertainties in antibiotic decision-making by prescribers that occur due to an array of complex organisational and cultural factors. Diversification of priorities between different specialties creates loopholes in the continuity of antibiotic care and treatment. Our review indicates that the transition of patients between wards, busy work environment, high workload, poor documentation and communication and reluctance of junior doctors to question senior colleagues contribute to the partial loss of ownership of antibiotic decisions.

The concept of antibiotic decision ownership does not appear to be highlighted by previous reviews of hospital antibiotic prescribing. It can be argued, however, that when health professionals have a sense of decision ownership, they become personally invested in clinical decisions made for their patients [47]. Although infection management and antibiotic decisions are inherently team-based and interprofessional in nature [48], findings from our ME show that stopping or de-escalating therapy is seen as the responsibility of the consultant or senior specialist. The disparity between expectations of junior clinicians to start but not review and/or stop antibiotics has been previously addressed in a realist review [49], which found that there is a lack of clarity around the specific roles and responsibilities that trainees undertake in relation to antimicrobial prescribing. Communicating an expectation for this group to gain active responsibility for prescribing decisions was suggested as a possible solution to overcome the issue. A recent observational study comparing antibiotic decision-making in acute medical and surgical specialties at a London teaching hospital found that the loss of ownership occurred in the transition of care between the emergency department and inpatient teams specifically [12]. Our ME findings confirm this is the case across different hospital settings and highlights the complexity that arises from each individual's responsibility for the collective problem of antimicrobial resistance being blurred.

Furthermore, this review has identified inconsistencies in the provision of information between specialties and healthcare professionals. The healthcare system heavily relies on the patient medical records for communication, and safe, and effective care as patients move between wards and their care is handed over between different clinical teams and when staff shifts change. Despite international efforts suggesting that clear documentation 
of decisions is a key principle in advancing patient safety and improving outcomes [7], the findings show poor documentation of decisions leading to unnecessary continuation of antibiotics as clinicians lack adequate information to make an appropriate decision whether to stop, continue or switch the treatment.

Some studies included in the review appear to indicate that with uncertainty, when an infection is suspected but not proven, the treating clinician will balance immediate clinical risks over long-term population risk. Although commencing antibiotics may be beneficial to the individual, excessive use can increase future AMR and thus be detrimental to the society, a situation known as 'the tragedy of the commons' [50]. Considering population implications of AMR within bedside antibiotic decisions was viewed by clinicians as difficult. To eliminate concrete clinical concerns, some clinicians will adopt their behaviour accordingly to the culturally accepted norms of the hospital and choose an activity that is perceived as low risk at an individual-level. This fear of consequences heightened by the perception that being conservative in prescribing is not seen as good practice will often lead to prescribing outside of clinical guidelines (either broader spectrum or for longer duration than is clinically indicated), without any clinical benefit to individual patients [18]. Driven by fear of patient deteriorating, an individual's capacity to adhere to evidence-based practice may be diminished and antibiotic optimisation becomes an absent priority, whilst the risks of over-prescribing to the individual patient tend to be downgraded. This dichotomy between the care recommended in the guidelines and the care provided at the bedside has been reported in earlier works as 'being on the safe side' [51].

The ME further highlights the interprofessional nature of antibiotic prescribing and the associated difficulties in negotiating decisions with multiple authoritative figures, including the immediate clinical team and other specialties. Discord in interpersonal relationships was an influencing factor on prescribing decisions, at times leading to poor continuity of care. Inconsistent advice and misunderstanding of roles and responsibilities pertaining to antibiotic decisions were additional barriers to successful collaboration. Challenging decisions of senior colleagues was perceived as unacceptable. The reluctance of junior doctors to question the prescribing decisions can act as an obstacle to gaining a clear understanding of why prescribing choices differ [49]. In such an environment, deferral to the opinion leaders can become the default mode of practice, suppressing valuable input from all members of the team. Yet, qualitative research suggests that doctors tend to feel drawn towards supportive teams and teachers who engage with or inspire them [52]. In environments where senior clinicians are approachable, trust in working relationships increases, allowing junior doctors to raise questions and thus close the communication gap [53, 54]. Examples of good practice included the presence of a clinical pharmacist, infectious disease and microbiology colleagues on the ward prompting the review of antibiotics and acting as effective communication channels.

A collaborative culture fostering a multidisciplinary approach and normalisation of the role of other specialists within the decision-making process are crucial to aid improvements to antimicrobial stewardship [55]. This review demonstrates that the involvement from other specialties in the decision-making depends on the familiarity and acceptance of those colleagues by the senior clinicians. Some junior doctors in these studies described managing interactions with other healthcare professionals as challenging. The 'unspoken' yet widely accepted rules on how to manage multidisciplinary dynamics mean that doctors face difficulties steering through the complex system of interrelationships with colleagues that could potentially provide them with assistance. Yet, turning to other specialties for advice can be a source of support outside the scope of medical hierarchy and the immediate clinical team, as junior clinicians experience less fear of appearing ignorant and attracting criticism $[56,57]$.

Although literature is still lacking with regards to the contexts under which junior doctors feel more able to challenge decisions effectively, quantitative evidence shows that the provision of feedback on the quality of prescribing and direct interaction with prescribers appear to have the most lasting impact on practice [58, 59]. A recent Cochrane review on interventions to improve antimicrobial prescribing practices for hospital inpatients found that interventions that included feedback were more effective than those that did not [8]. Findings of the ME complement the review by showing that creating effective feedback mechanisms and improving communication on prescribing practice has a potential to elicit behavioural change.

In addition, endorsements for the greater integration of other prescribing groups, including pharmacists and nurse prescribers within antibiotic stewardship efforts have already been highlighted by others [60]. For example, lack of partnership with nurses can limit the success of antibiotic stewardship initiatives [61]. Yet, this ME identified an absence of perceived or reported nursing involvement in antibiotic decision-making. This may reflect perceptions about antibiotic prescribing as a process that requires increased knowledge only exclusive to medical professionals with prescribing powers [62], existing and gaps in undergraduate and postgraduate education about antibiotics and AMR [63]. Yet, it remains essential to maximise the contribution of the existing professionals outside infection disease and 
microbiology towards appropriate use of antimicrobials [64], especially in view of the new Nursing and Midwifery Council guidance highlighting that newly qualified nurses have to be prepared to undertake prescribing training soon after registration [65].

Lastly, antimicrobial prescribing behaviours may vary significantly across different hospital types and be influenced by the types of patients admitted, prescribing patterns and the resources available. For example, a previous study using data gathered from a nationwide survey highlighted major differences in the available resources and implementation of AMS programmes between public and private hospitals in Australia [66]. Moreover, significant differences in antibiotic use remain across different hospital types. In adjusted models, teaching hospitals were associated with lower use of third- and fourth-generation cephalosporins and antipseudomonal agents [67]. Although doctors working in private hospitals acknowledged treating more 'aggressively' with broader-spectrum antibiotics when patient follow-up was uncertain [36], no major sector-specific drivers of doctors ' prescribing behaviour emerged in the synthesis. These findings suggest that antibiotic prescribing across different countries and healthcare systems may be influenced by a similar set of cultural factors [37]. However, given that most studies included in this synthesis were conducted in public teaching hospitals, such as in the UK, the developed model can only be claimed to be representative of that context.

\section{Strengths and limitations}

Locating suitable qualitative studies can be challenging [68] and small-scale qualitative research can be perceived as biased and lacking transferability [69]. However, the number of included studies in the synthesis $(n=15)$ from seven countries and reflecting a breadth of prescribing perspectives was sufficient for conducting ME [20]. The synthesis was carried out in a rigorous way including a large range of databases and grey literature, with continuous input from the experienced research team, undoubtedly reinforcing the credibility of the findings. All stages of the review were checked for accuracy and were grounded in the data by constantly checking the findings against the original studies.

The novelty of this ME is the generation of a higher translation that helps to understand the complexities of hospital antibiotic prescribing decision-making. Although the conceptual model cannot be claimed to be definitive and represent all healthcare practitioners, it offers a unique lens, through which the experiences of doctors can be considered. Meta-ethnography is an interpretative approach and the development of the conceptual model was informed by the review team`s backgrounds and perspectives. The team had considerable expertise in synthesising qualitative research, including experienced health professionals and social scientists with an interest and experience in developing behaviour-change interventions, but none were medical prescribers. GW had been previously involved in projects exploring hospital antibiotic stewardship, NR and $\mathrm{BW}$ have extensive experience in conducting ME and $\mathrm{NR}$ is a co-author of the eMERGe metaethnography reporting guidance. We conducted our research in close affiliation with an NHS hospital trust with advisory input from clinicians during the project. We acknowledge that a different team may have interpreted the included studies differently.

There is currently no gold standard of appraising qualitative studies and including studies with poorly reported methods could produce ME findings lacking credibility [26]. We conducted critical appraisal using the CASP tool, but a different approach of judging the 'weight of evidence' of each paper may have been justified. To be included in our synthesis, studies needed to meet a certain degree of methodological transparency. This decision was appropriate as there was a large number of methodologically transparent eligible studies that we could review, and which would enable us to rigorously develop new interpretations and an LOA. After creating our LOA we compared our interpretation and findings against the papers excluded following quality appraisal $[29,31]$. This strategy ensured that important insights have not been missed, thus eliminating potential bias. One study raised an issue relating to senior doctors ' perception that inappropriate antibiotic prescribing outside guideline recommendations originates with junior doctors [29]. Although this perception did not feature in our analysis, including this paper would not have changed the final synthesis. To enhance the quality of the ME, we updated our database searches in December 2020 and found five studies that met our inclusion criteria [12, 70-73]. However, on critically reading them, we believe that including these studies in our ME would have not refuted our findings but resulted in equivalent meaning.

Not all included studies reported details of participants` characteristics, including gender, ethnicity, level of training, length of experience, and some studies analysed data together for samples drawn from across different clinical settings and healthcare systems. Therefore, it was not always possible to fully identify disconfirming cases between papers or carry out a subanalysis of different drivers of behaviour based on the sample characteristics and study context. Additionally, five included papers were published by the same researchers research team and although the authors explored prescribing practices in two different countries 
(Australia and UK), the results may have inadvertently influenced our findings and synthesis [34, 40-42, 45].

The exclusion of studies describing views and experiences of healthcare professionals other than doctors, or where the study population included a mix of healthcare professionals may be contested and a more inclusive approach exploring more diverse perceptions across different clinical groups may have been warranted. However, given that the majority of antibiotics are prescribed by doctors, it was vital to first understand their views and experiences of prescribing practice. The decision was also made to exclude low-income countries to ensure that the theory generated from synthesising primary studies reflects the function of $\mathrm{ME}$ and is relevant to the context and setting of the planned antibiotic intervention, that is acute hospitals in well-developed healthcare systems. Including relatively homogenous studies helped strengthen the weight of the conceptual model.

Finally, to increase the credibility of the review and ensure that the breadth and scope of the data were captured in the synthesis, findings were critically reflected on through regular briefing sessions and workshops with key stakeholders (healthcare professionals involved in hospital antimicrobial stewardship and health service users), providing opportunities to develop and refine ideas and interpretations, and analysed using multiple theoretical perspectives. Although decontextualisation of qualitative findings can be debated [74], the quality and rigour of this review means that it is possible to apply the new conceptual model to a variety of clinical contexts and different groups of healthcare professionals.

\section{Future practice and research implications}

This ME highlights that there is a need to incorporate the influence of the micro- and macro-level elements in the design and delivery of future behavioural-change interventions to optimise antibiotic use in hospital settings. Addressing this complex interaction may be a contributing factor to finding future solutions to the ever-growing problem of AMR and reducing fear of consequences from non-prescribing or stopping antibiotics. Finding new ways of discussing and questioning prescribing decisions between and within clinical teams may be one strategy to mitigate the negative impact of the loss of ownership of decisions and reduce failures in the provision of adequate information. In clinical practice, the influence of senior colleagues could be harnessed by creating role models who act as custodians of professional agendas and create a supportive and open environment that fosters the culture of learning and feedback. The high-level findings presented in this analysis could be further developed for implementation in practice. The insights into ' doctors 'conceptualisation of antibiotic use could also have implications for behavioural interventions in other settings, such as primary care or long-term facilities.

The findings in this study concerning the loss of decision ownership may be worth further empirical examination, with a large sample and across a diverse population. It is suggested that future research about promoting effective hospital antimicrobial stewardship focuses on exploring the idea of invisibility of prescribing decisions. Specifically, it would be of value to investigate the diversity of opinions around the roles and responsibilities junior prescribers should undertake in relation to antimicrobial prescribing and how to help overcome uncertainty and fear of consequences. Finding ways to communicate an expectation for this group may foster transfer of active responsibility down the hierarchy ladder. LastlyMoreover, there remains a gap in research concerning the contexts under which junior doctors feel more able to challenge seniors' decisions effectively. Lastly, identifying and comparing inter-hospital factors associated with inappropriate prescribing across different sectors (private vs. public, teaching vs. non-teaching) will help direct future AMS efforts in the specific settings. These areas warrant further investigation.

\section{Conclusions}

This novel ME extends the current evidence-base by providing an understanding of the complexities of hospital antibiotic prescribing. The resulting conceptual framework has the potential to act as the basis for future antibiotic management interventions, exploring clinicians 'internal logic of antibiotic prescribing behaviours that goes beyond antimicrobial guidelines and evidencebased practice. Changing ingrained behaviours within a culture or an organisation is undeniably difficult. Yet, improving prescribing practices is essential to minimising the growing public health threat of AMR. It is particularly challenging in acute hospital settings due to the complex relationships between a wide range of stakeholders and multiple teams. Acknowledging this complexity and variability of the hospital contexts and recognising the norms and the ways in which doctors learn to practice will facilitate that change. Healthcare stakeholders can draw on this evidence of how and why doctors make prescribing decisions to help design and implement more effective antibiotic stewardship interventions in secondary care.

Finally, uncertainty is an unavoidable part of clinical practice and will inevitably persist across all spheres of medicine. Thus, the key dilemma for policymakers and healthcare providers is how to place a higher value on non-prescribing or prescribing narrow-spectrum antibiotics, when available and efficacious, and eliminate a degree of fear while making decisions under uncertain conditions. This ME highlights the need for a more 
collaborative culture fostering normalisation of the role of other specialists within the decision-making process. The quality of inter-professional relationships between clinicians remains key to achieving this change. Reclaiming the 'why' may act as a positive force to shift the individual risk perceptions and have a positive knock-on effect on changing the culture to open collaboration. This shift will require engagement from senior colleagues, managers and opinion leaders to acknowledge the importance of maximising the explanatory knowledge acquisition.

\section{Abbreviations}

AMR: Antimicrobial resistance; AMS: Antimicrobial stewardship; CASP: Critical Appraisal Skills Programme; HCC: Higher conceptual category; LOA: Line-ofargument; ME: Meta-ethnography; PRISMA: Preferred Reporting Items for Systematic Reviews and Meta-Analyses; SPIDER: Sample, Phenomenon of Interest, Design, Evaluation, Research type; UK: United Kingdom; US: United States

\section{Supplementary Information}

The online version contains supplementary material available at https://doi. org/10.1186/s13690-021-00624-1.

Additional file 1. Details of applied methodology as informed by the eMERGe meta-ethnography reporting guidance.

Additional file 2. Relating studies by reported concepts and developing higher conceptual categories (Phase 4).

Additional file 3. Key emerging themes with exemplar quotes.

\section{Acknowledgements}

We would like to thank Sheena Moffat, the University Librarian, for her assistance in carrying out database searches for the review.

\section{Authors' contributions}

All authors made substantive contributions to the qualitative synthesis reported in this paper. GW and CM conducted the searches and the critical appraisal of included studies. GW, NR and KK provided critical input on the design and analysis of the ME and made substantive contributions to manuscript drafts. GW drafted the manuscript and all authors commented on drafts and approved the final manuscript.

\section{Funding}

This review was carried out as part of a PhD by the first author funded by the Edinburgh Napier University.

\section{Availability of data and materials}

This systematic review is based on an analysis of a number of published papers which are all referenced within this manuscript. Data supporting our findings is included in the form of the supplementary files listed below.

\section{Declarations}

Ethics approval and consent to participate

Not applicable.

\section{Consent for publication}

Not applicable.

\section{Competing interests}

The authors declare that they have no competing interests.

\section{Author details}

${ }^{1}$ School of Health and Social Care, Edinburgh Napier University, EH11 4BN Edinburgh, UK. ${ }^{2}$ Edinburgh Critical Care Research Group, University of Edinburgh, Edinburgh Royal Infirmary, EH16 4SA Edinburgh, UK.

Received: 1 February 2021 Accepted: 30 May 2021

Published online: 23 July 2021

\section{References}

1. Tacconelli E, Pezzani MD. Public health burden of antimicrobial resistance in Europe. Lancet Infect Dis. 2019;19:4-6. https://doi.org/10.1016/S1473-3099(1 8)30648-0.

2. Barber S, Swaden-Lewis K Briefing Paper, Antimicrobial Resistance - House of Commons Library. Briefing Paper. 2017. https://commonslibrary.parlia ment.uk/research-briefings/cbp-8141/. Accessed 19 Aug 2019.

3. European Centre for Disease Prevention and Control. Surveillance of antimicrobial resistance in Europe Annual report of the European Antimicrobial Resistance Surveillance Network (EARS-Net) 2018. 2019. https://doi.org/10.2900/230516.

4. Klein EY, Van Boeckel TP, Martinez EM, Pant S, Gandra S, Levin SA, et al. Global increase and geographic convergence in antibiotic consumption between 2000 and 2015. Proc Natl Acad Sci USA. 2018:115:E3463-70. https://doi.org/10.1073/pnas.1717295115.

5. Luepke KH, Suda KJ, Boucher H, Russo RL, Bonney MW, Hunt TD, et al. Past, Present, and Future of Antibacterial Economics: Increasing Bacterial Resistance, Limited Antibiotic Pipeline, and Societal Implications. Pharmacotherapy. 2017;37:71-84. https://doi.org/10.1002/phar.1868.

6. O'Neill J. Antimicrobial Resistance: Tackling a crisis for the health and wealth of nations. Rev Antimicrob Resist. 2016. https://amr-review.org/sites/default/ files/AMR\%20Review\%20Paper\%20-\%20Tackling\%20a\%20crisis\%20for\%2 0the\%20health\%20and\%20wealth\%20of\%20nations_1.pdf. Accessed 03 May 2018

7. Centre for Disease Control and Prevention. Core Elements of Hospital Antibiotic Stewardship Programs is a publication of The National Center for Emerging and Zoonotic Infectious Diseases within the Centers for Disease Control and Prevention. 2019. https://www.cdc.gov/antibiotic-use/healthca re/pdfs/hospital-core-elements-H.pdf Accessed 7 Sep 2019.

8. Davey P, Marwick CA, Scott CL, Charani E, McNeil K, Brown E, et al. Interventions to improve antibiotic prescribing practices for hospital inpatients. Cochrane Database Syst Rev. 2017;2(2):CD003543. https://doi. org/10.1002/14651858.CD003543.pub4.

9. Public Health England. English surveillance programme for antimicrobial utilisation and resistance (ESPAUR). Report 2018-2019. https://assets. publishing.service.gov.uk/government/uploads/system/uploads/attachment data/file/843129/English_Surveillance_Programme_for_Antimicrobial. 2019. https://assets.publishing.service.gov.uk/government/uploads/system/uploa ds/attachment_data/file/843129/English_Surveillance_Programme_for_A ntimicrobial. 2019. Accessed 11 June 2019.

10. Craig P, Dieppe P, Macintyre S, Michie S, Nazareth I, Petticrew M. Developing and evaluating complex interventions: new guidance. BMJ. 2008;337:a1655. https://doi.org/10.1136/bmj.a1655.

11. Hulscher ME, Prins JM. Antibiotic stewardship: does it work in hospital practice? A review of the evidence base. Clin Microbiol Infect. 2017;23:799805. https://doi.org/10.1016/j.cmi.2017.07.017.

12. Charani E, Ahmad R, Rawson TM, Castro-Sanchèz E, Tarrant C, Holmes AH. The differences in antibiotic decision-making between acute surgical and acute medical teams: An ethnographic study of culture and team dynamics. Clin Infect Dis. 2019;69:12-20. https://doi.org/10.1093/cid/ciy844.

13. Nielsen K, Miraglia M. What works for whom in which circumstances? On the need to move beyond the 'what works?' question in organizational intervention research. Hum Relations. 2017;70:40-62. https://doi.org/10.11 77/0018726716670226

14. Lorencatto F, Charani E, Sevdalis N, Tarrant C, Davey P. Driving sustainable change in antimicrobial prescribing practice: How can social and behavioural sciences help? J Antimicrob Chemother. 2018;73:2613-24 https://doi.org/10.1093/jac/dky222.

15. Noyes J. Never mind the qualitative feel the depth! The evolving role of qualitative research in Cochrane intervention reviews. J Res Nurs. 2010;15: 525-34. https://doi.org/10.1177/1744987110381696.

16. Dixon-Woods M, Cavers D, Agarwal S, Annandale E, Arthur A, Harvey J, et al. Conducting a critical interpretive synthesis of the literature on access to 
healthcare by vulnerable groups. BMC Med Res Methodol. 2006;6:35 https://doi.org/10.1186/1471-2288-6-35.

17. Tonkin-Crine S, Yardley L, Little P. Antibiotic prescribing for acute respiratory tract infections in primary care: a systematic review and meta-ethnography. J Antimicrob Chemother. 2011;66:2215-23. https://doi.org/10.1093/jac/dkr2 79.

18. Krockow E, Colman A, Chattoe E, Jenkins R, Perera D, Mehtar N. S, et al. Balancing the risks to individual and society: A systematic review and synthesis of qualitative research on antibiotic prescribing behaviour in hospitals. J Hosp Infect. 2018;101(4):428-39. https://doi.org/10.1016/j.jhin.201 8.08.007.

19. France EF, Ring N, Thomas R, Noyes J, Maxwell M, Jepson R. A methodological systematic review of what's wrong with meta-ethnography reporting. BMC Med Res Methodol. 2014;14:119. https://doi.org/10.1186/14 71-2288-14-119.

20. Campbell R, Pound P, Morgan M, Daker-White G, Britten N, Pill R, et al. Evaluating meta-ethnography: systematic analysis and synthesis of qualitative research. Health Technol Assess. 2011;15:1-164. https://doi.org/1 0.3310/hta15430.

21. Noblit GW, Hare RD. Meta-ethnography: Synthesizing qualitative studies. California: Sage Publications Ltd; 1988. https://doi.org/10.1097/00005053-1 99007000-00016

22. France EF, Cunningham M, Ring N, Uny I, Duncan EAS, Jepson RG, et al. Improving reporting of meta-ethnography: The eMERGe reporting guidance. BMC Med Res Methodol. 2019;19:25. https://doi.org/10.1186/s12 874-018-0600-0.

23. Cooke A, Smith D, Booth A. Beyond PICO. The SPIDER Tool for Qualitative Evidence Synthesis. Qual Health Res. 2012;22:1435-43. https://doi.org/10.11 77/1049732312452938.

24. DeJean D, Giacomini M, Simeonov D, Smith A. Finding Qualitative Research Evidence for Health Technology Assessment. Qual Health Res. 2016;26: 1307-17. https://doi.org/10.1177/1049732316644429.

25. Critical Appraisal Skills Programme. Qualitative Checklist CASP. Casp 2018. https://casp-uk.net/wp-content/uploads/2018/03/CASP-QualitativeChecklist-2018_fillable_form.pdf Accessed 21 Feb 2018.

26. Toye F, Seers K, Allcock N, Briggs M, Carr E, Barker K. Meta-ethnography 25 years on: challenges and insights for synthesising a large number of qualitative studies. BMC Med Res Methodol. 2014;14:80. https://doi.org/10.11 86/1471-2288-14-80

27. Toye F, Seers K, Barker KL. Meta-ethnography to understand healthcare professionals' experience of treating adults with chronic non-malignant pain. BMJ Open. 2017;7:e018411. https://doi.org/10.1136/bmjopen-2017-018411.

28. Britten N, Campbell R, Pope C, Donovan J, Morgan MY, Pill R. Using meta ethnography to synthesise qualitative research: a worked example. J Health Serv Res Policy. 2002;7:209-15.

29. Almatar MA, Peterson GM, Thompson A, Zaidi STR. Factors influencing ceftriaxone use in community-acquired pneumonia: Emergency doctors' perspectives. Emerg Med Australas. 2014;26:591-5. https://doi.org/10.1111/1 742-6723.12326

30. Almatar MA. Implementation and evaluation of tailored intervention strategies to influence antibiotic prescribing for community-acquired pneumonia. PHD Thesis. University of Tasmania; 2015. ePrints. https:// eprints.utas.edu.au/22746/.

31. Barlow G, Nathwani D, Myers E, Sullivan F, Stevens N, Duffy R, et al. Identifying barriers to the rapid administration of appropriate antibiotics in community-acquired pneumonia. J Antimicrob Chemother. 2008;61:442-51.

32. Cortoos P-J, De Witte K, Peetermans WE, Simoens S, Laekeman G. Opposing expectations and suboptimal use of a local antibiotic hospital guideline: a qualitative study. J Antimicrob Chemother. 2008;62:189-95. https://doi.org/1 0.1093/jac/dkn143

33. Bjorkman I, Berg J, Roing M, Erntell M, Lundborg CS. I. B, et al. Perceptions among Swedish hospital physicians on prescribing of antibiotics and antibiotic resistance. Qual Safety in Health Care. 2010;19(6):e8. https://doi. org/10.1136/qshc.2008.029199.

34. Broom A, Broom J, Kirby E. Cultures of resistance? A Bourdieusian analysis of doctors' antibiotic prescribing. Soc Sci Med. 2014;110:81-8. https://doi.org/1 0.1016/j.socscimed.2014.03.030.

35. Mattick K, Kelly N, Rees C. A window into the lives of junior doctors: narrative interviews exploring antimicrobial prescribing experiences. J Antimicrob Chemother. 2014;69:2274-83. https://doi.org/10.1093/jac/ dku093.
36. May L, Gudger G, Armstrong P, Brooks G, Hinds P, Bhat R, et al. Multisite exploration of clinical decision making for antibiotic use by emergency medicine providers using quantitative and qualitative methods. Infect Control Hosp Epidemiol. 2014;35:1114-25. https://doi.org/10.1086/677637.

37. Livorsi D, Comer A, Matthias MS, Perencevich EN, Bair MJ. Factors Influencing Antibiotic-Prescribing Decisions Among Inpatient Physicians: A Qualitative Investigation. Infect Control Hosp Epidemiol. 2015;36:1065-72. https://doi.org/10.1017/ice.2015.136.

38. Livorsi D, Comer AR, Matthias MS, Perencevich EN, Bair MJ. Barriers to guideline-concordant antibiotic use among inpatient physicians: A case vignette qualitative study. J Hosp Med. 2016;11:174-80. https://doi.org/10.1 002/jhm.2495.

39. Skodvin B, Aase K, Charani E, Holmes A, Smith I, et al. An antimicrobial stewardship program initiative: a qualitative study on prescribing practices among hospital doctors. Antimicrob Resist Infect Control. 2015:4:24. https://doi.org/10.1186/s13756-015-0065-4.

40. Broom J, Broom A, Adams K, Plage S. What prevents the intravenous to oral antibiotic switch? A qualitative study of hospital doctors' accounts of what influences their clinical practice. J Antimicrob Chemother. 2016;71:2295-9. https://doi.org/10.1093/jac/dkw129.

41. Broom J, Broom A, Plage S, Adams K, Post JJ. Barriers to uptake of antimicrobial advice in a UK hospital: a qualitative study. J Hosp Infect. 2016; 93:418-22. https://doi.org/10.1016/j.jhin.2016.03.011.

42. Broom A, Broom J, Kirby E, Adams J. The social dynamics of antibiotic use in an Australian hospital. J Sociol. 2016;52:824-39. https://doi.org/10.1177/144 0783315594486

43. Eyer MM, Lang M, Aujesky D, Marschall J. Overtreatment of asymptomatic bacteriuria: a qualitative study. J Hosp Infect. 2016;93:297-303. https://doi. org/10.1016/j.jhin.2016.04.007.

44. Rawson TM, Charani E, Moore LSP, Hernandez B, Castro-Sanchez E, Herrero $P$, et al. Mapping the decision pathways of acute infection management in secondary care among UK medical physicians: a qualitative study. BMC Med. 2016;14:208. https://doi.org/10.1186/s12916-016-0751-y.

45. Broom J, Broom A, Kirby E, Gibson AF, Post JJ. Individual care versus broader public health: A qualitative study of hospital doctors' antibiotic decisions. Infect Dis Heal. 2017;22:97-104. https://doi.org/10.1016/jiidh.2017. 05.003.

46. Sedrak A, Anpalahan M, Luetsch K. Enablers and barriers to the use of antibiotic guidelines in the assessment and treatment of communityacquired pneumonia_A qualitative study of clinicians' perspectives. Int J Clin Pract. 2017;71:e12959. https://doi.org/10.1111/ijcp.12959.

47. Dubov A, Fraenkel L, Seng E. The Importance of Fostering Ownership During Medical Training. Am J Bioeth. 2016;16:3-12. https://doi.org/10.1 080/15265161.2016.1197338.

48. Broom A, Broom J, Kirby E, Plage S, Adams J. What role do pharmacists play in mediating antibiotic use in hospitals? A qualitative study. BMJ Open. 2015;5:e008326. https://doi.org/10.1136/bmjopen-2015-008326.

49. Papoutsi C, Mattick K, Pearson M, Brennan N, Briscoe S, Wong G. Interventions to improve antimicrobial prescribing of doctors in training (IMPACT): a realist review. Heal Serv Deliv Res. 2018;6:1-136. https://doi. org/10.3310/hsdr06100.

50. Hardin G. The Tragedy of the Commons. Science. 1968;162(3859):1243-8.

51. Walker S, McGeer A, Simor AE, Armstrong-Evans M, Loeb M. Why are antibiotics prescribed for asymptomatic bacteriuria in institutionalized elderly people? A qualitative study of physicians' and nurses' perceptions. CMAJ. 2000;163:273-7.

52. Spooner S, Pearson E, Gibson J, Checkland K. How do workplaces, working practices and colleagues affect UK doctors' career decisions? A qualitative study of junior doctors' career decision making in the UK. BMJ Open. 2017; 7. https://doi.org/10.1136/bmjopen-2017-018462.

53. Bould MD, Sutherland S, Sydor DT, Naik V, Friedman Z. Residents' reluctance to challenge negative hierarchy in the operating room: a qualitative study. Can J Anesth. 2015;62:576-86. https://doi.org/10.1007/s12630-015-0364-5.

54. Friedman Z, Hayter MA, Everett TC, Matava CT, Noble LMK, Bould MD. Power and conflict: the effect of a superior's interpersonal behaviour on trainees' ability to challenge authority during a simulated airway emergency. Anaesthesia. 2015;70:1119-29. https://doi.org/10.1111/anae.13191.

55. Chaves NJ, Cheng AC, Runnegar N, Kirschner J, Lee T, Buising K. Analysis of knowledge and attitude surveys to identify barriers and enablers of appropriate antimicrobial prescribing in three Australian tertiary hospitals. Intern Med J. 2014;44:568-74. 
56. Papoutsi C, Mattick K, Pearson M, Brennan N, Briscoe S, Wong G. Social and professional influences on antimicrobial prescribing for doctors-in-training: a realist review. J Antimicrob Chemother. 2017;72:2418-30. https://doi.org/1 0.1093/jac/dkx194

57. Tallentire VR, Smith SE, Skinner J, Cameron HS. Understanding the behaviour of newly qualified doctors in acute care contexts. Med Educ. 2011:45:995-1005. https://doi.org/10.1111/j.1365-2923.2011.04024.x.

58. Hersh AL, Beekmann SE, Polgreen PM, Zaoutis TE, Newland JG. Antimicrobial Stewardship Programs in Pediatrics. Infect Control Hosp Epidemiol. 2009;30:1211-7. https://doi.org/10.1086/648088.

59. Wagner B, Filice GA, Drekonja D, Greer N, MacDonald R, Rutks I, et al. Antimicrobial Stewardship Programs in Inpatient Hospital Settings: A Systematic Review. Infect Control Hosp Epidemiol. 2014;35:1209-28. https://doi.org/10.1086/678057.

60. Olans RD, Olans RN, Witt DJ. Good Nursing Is Good Antibiotic Stewardship. Am J Nurs. 2017;117(8):58-63. https://doi.org/10.1097/01.NAJ.0000521974. 76835.e0.

61. Carter EJ, Greendyke WG, Yoko Furuya E, Srinivasan A, Shelley AN, Bothra A, et al. Exploring the nurses' role in antibiotic stewardship: A multisite qualitative study of nurses and infection preventionists. 2018;46(5):492-497. https://doi.org/10.1016/j.ajic.2017.12.016.

62. Castro-Sánchez E, Bennasar-Veny M, Smith M, Singleton S, Bennett E, Appleton J, et al. European Commission guidelines for the prudent use of antimicrobials in human health: a missed opportunity to embrace nursing participation in stewardship. Clin Microbiol Infect. 2018;24:914-5. https://doi. org/10.1016/..cmi.2018.02.030

63. Rawson TM, Butters TP, Moore LSP, Castro-Sánchez E, Cooke FJ, Holmes AH. Exploring the coverage of antimicrobial stewardship across UK clinical postgraduate training curricula. J Antimicrob Chemother. 2016;71:3284-92.

64. Edwards R, Drumright L, Kiernan M, Holmes A. Covering more territory to fight resistance: Considering nurses' role in antimicrobial stewardship. J Infect Prev. 2011;12(1):6-10. https://doi.org/10.1177/1757177410389627.

65. Nursing and Midwifery Council. Realising professionalism: Standards for education and training Part 1: Standards framework for nursing and midwifery education. 2018. https://www.nmc.org.uk/standards-for-educa tion-and-training/standards-framework-for-nursing-and-midwifery-educa tion/ Accessed 09 Sep 2020.

66. Cotta MO, Chen C, Tacey M, James RS, Buising KL, Marshall C, et al. What are the similarities and differences in antimicrobial prescribing between Australian public and private hospitals? Intern Med J. 2016;46(10):1182-8. https://doi.org/10.1111/imj.13209.

67. Goodman KE, Cosgrove SE, Pineles L, Magder LS, Anderson DJ, Dodds Ashley E, et al. Significant Regional Differences in Antibiotic Use Across 576 US Hospitals and 11,701,326 Million Adult Admissions, 2016-2017. Clin Infect Dis. 2020;ciaa570. https://doi.org/10.1093/cid/ciaa570.

68. Booth A. Searching for qualitative research for inclusion in systematic reviews: A structured methodological review. Syst Rev. 2016;5:74. https://doi. org/10.1186/s13643-016-0249-x.

69. Trochim WMK, Donnelly JP, Arora K. Research methods: the essential knowledge base. Canada: Nelson Education Ltd; 2016.

70. Broom J, Broom A, Kirby E, Post JJ. Improvisation versus guideline concordance in surgical antibiotic prophylaxis: a qualitative study. Infection. 2018;46(4):541-8. https://doi.org/10.1007/s15010-018-1156-y.

71. Kajamaa A, Mattick K, Parker H, Hilli A, Rees C. Trainee doctors' experiences of common problems in the antibiotic prescribing process: An activity theory analysis of narrative data from UK hospitals. BMJ Open. 2019;9: e028733. https://doi.org/10.1136/bmjopen-2018-028733.

72. Morgan JR, Barlam TF, Drainoni ML. A Qualitative Study of the Real-world Experiences of Infectious Diseases Fellows Regarding Antibiotic Stewardship. Open Forum Infect Dis. 20185(9):ofy102. https://doi.org/10.1093/ofid/ofy102.

73. Broom JK, Broom AF, Kirby ER, Post JJ. How do professional relationships influence surgical antibiotic prophylaxis decision making? A qualitative study. Am J Infect Control. 2018;43(3):311-5. https://doi.org/10.1016/j.ajic.2017.09.004.

74. Friberg F, Dahlberg K, Petersson MN, Öhlén J. Context and methodological decontextualization in nursing research with examples from phenomenography. Scand J Caring Sci. 2000;14:37-43. https://doi.org/1 0.1111/j.1471-6712.2000.tb00559.x.

\section{Publisher's Note}

Springer Nature remains neutral with regard to jurisdictional claims in published maps and institutional affiliations.

Ready to submit your research? Choose BMC and benefit from:

- fast, convenient online submission

- thorough peer review by experienced researchers in your field

- rapid publication on acceptance

- support for research data, including large and complex data types

- gold Open Access which fosters wider collaboration and increased citations

- maximum visibility for your research: over $100 \mathrm{M}$ website views per year

At BMC, research is always in progress.

Learn more biomedcentral.com/submissions 